\title{
Close interpersonal proximity modulates visuomotor processing of object affordances in shared, social space
}

\author{
Elizabeth J. Saccone ${ }^{1,2} \cdot$ Ancret Szpak $^{3}$ - Owen Churches ${ }^{1} \cdot$ Michael E. R. Nicholls $^{1}$
}

Published online: 1 September 2017

(C) The Psychonomic Society, Inc. 2017

\begin{abstract}
Research suggests that the human brain codes manipulable objects as possibilities for action, or affordances, particularly objects close to the body. Near-body space is not only a zone for body-environment interaction but also is socially relevant, as we are driven to preserve our near-body, personal space from others. The current, novel study investigated how close proximity of a stranger modulates visuomotor processing of object affordances in shared, social space. Participants performed a behavioural object recognition task both alone and with a human confederate. All object images were in participants' reachable space but appeared relatively closer to the participant or the confederate. Results revealed when participants were alone, objects in both locations produced an affordance congruency effect but when the confederate was present, only objects nearer the participant elicited the effect. Findings suggest space is divided between strangers to preserve independent near-body space boundaries, and in turn this process influences motor coding for stimuli within that social space. To demonstrate that this visuomotor modulation represents a social phenomenon, rather than a general, attentional effect, two subsequent experiments employed
\end{abstract}

Electronic supplementary material The online version of this article (https://doi.org/10.3758/s13414-017-1413-7) contains supplementary material, which is available to authorized users.

Elizabeth J. Saccone

elizabeth.saccone@ flinders.edu.au

1 College of Education, Psychology and Social Work, Flinders University, GPO Box 2100, Adelaide, SA 5001, Australia

2 School of Psychological and Public Health, La Trobe University, Edwards Road, Flora Hill, Bendigo, VIC 3552, Australia

3 Flinders Centre for Science Education in the 21st Century, Flinders University, GPO Box 2100, Adelaide, SA 5001, Australia nonhuman joint conditions. Neither a small, Japanese, waving cat statue (Experiment 2) nor a metronome (Experiment 3) modulated the affordance effect as in Experiment 1. These findings suggest a truly social explanation of the key interaction from Experiment 1. This study represents an important step toward understanding object affordance processing in real-world, social contexts and has implications broadly across fields of social action and cognition, and body space representation.

Keywords Personal space $\cdot$ Interpersonal proximity $\cdot$ Object affordance $\cdot$ Social space $\cdot$ Social cognition $\cdot$ Peripersonal space

\section{Introduction}

The physical and social world in which we exist is complex and dynamic, yet we navigate it with remarkable ease. Our rapid, unconscious decision making and motor precision translates to an extraordinary ability to interact with the environment. Without conscious thought, we can effortlessly carry food on a fork to our mouths or transport our bodies through a crowded marketplace, avoiding collisions with the people and objects around us. To account for our remarkable ability to interact with the physical environment, modern cognitive neuroscience research has investigated how the human brain represents objects in the space around our bodies.

Drawing from Gibson's (1979) theory that we directly perceive action afforded by the environment, object perception research suggests that the brain codes objects as possibilities for action, or affordances. For example, a cup affords grasping and a chair affords sitting. In support of affordance views, there is evidence demonstrating that simply attending to a highly manipulable object can activate related motor 
programs, even without conscious intention to act on it (Cardellicchio, Sinigaglia, \& Costantini, 2011; Chao \& Martin, 2000; Gerlach, Law, \& Paulson, 2002; Grèzes \& Decety, 2002; Tucker \& Ellis, 1998). Findings from Witt and colleagues have shown that our ability to act on an object influences our reported experience of it (Philbeck \& Witt, 2015; Witt, Linkenauger, \& Wickens, 2015 for reviews). These findings highlight the tight link between action processes and object perception - the idea that we perceive action possibilities, or affordances, in the physical environment.

Objects are not the only action-relevant stimuli in the environment; however, humans are highly social and the other people around us affect how we move our bodies and interact with the physical world. Others can afford us possibilities for action that would not otherwise be available. For instance, two people can work together to perform a task that an individual acting alone could not achieve, such as carrying a heavy table (Pezzulo, Iodice, Ferraina, \& Kessler, 2013; Richardson, Marsh, \& Baron, 2007). The others around us also influence our motor planning and behaviour even when we are acting independently. For example, we account for the presence of others when simply walking down a street (Soper \& Karasik, 1977) and adjust our gestures during speech (Özyürek, 2002) based on the location of others.

Given that both the objects and people around us drive our motor plans and behaviour, there is surprisingly little research into how these two types of action-relevant stimuli interact in their effect on our related perceptual and motor processes. Object affordance research is typically conducted on participants in isolation, in keeping with the tradition of experimental psychology to keep extraneous variables to a minimum. There has been a recent trend to include social variables in research into action processes, but these studies often have investigated how participant pairs coordinate to perform a collaborative, joint task (Ciardo, Lugli, Nicoletti, Rubichi, \& Iani, 2016; Sebanz, Bekkering, \& Knoblich, 2006). Research suggests that we recognise and represent the action possibilities of other people (Creem-Regehr, Gagnon, Geuss, \& Stefanucci, 2013 for a relevant review), but once again these studies typically focus on how these processes facilitate coordinated action. Indeed, in our highly social world we frequently act in cooperation with other people; other times we act despite others. Therefore, an important consideration remains: how the presence of other actors around us affects how we process affordances in the physical environment.

Although the impact of social proximity on affordance perception is largely unknown, there is evidence that other people influence our motor behaviour because of the ownership that we claim over our near-body space. We typically view the space immediately surrounding our bodies as our own private or personal space (Hall, 1966) and often alter our behaviour to maintain a comfortable distance from other people, for example, when choosing a seat on public transit or opting to stand instead (Evans \& Wener, 2007; Hirsch \& Thompson, 2011). Unwelcome personal space invasions often are associated with discomfort and physiological arousal (Evans \& Wener, 2007; Perry, Rubinsten, Peled, \& Shamay-Tsoory, 2013). Also, our desire to maintain a certain distance from other people, particularly strangers, likely serves an adaptive function in the interest of protecting our bodies from unwanted physical contact (de Vignemont \& Iannetti, 2015; Graziano $\&$ Cooke, 2006). Research also suggests that we are aware of and represent the near-body space of other people (Brozzoli, Gentile, Bergouignan, \& Ehrsson, 2013; Maister, Cardini, Zamariola, Serino, \& Tsakiris, 2015), which may contribute to maintaining comfortable social distances.

Near-body space is not only socially relevant; it is also the region in which we are most likely to interact with objects in the physical environment. In fact, cognitive neuroscience has provided evidence that the brain uniquely represents this space by its potential for action and interaction with the physical world (Fogassi et al., 1996; Graziano \& Gross, 1994; Rizzolatti, Fadiga, Fogassi, \& Gallese, 1997). This practical, motor-based representation of close, actionable space, known in the neuroscience literature as peripersonal space (Rizzolatti et al., 1997), is neurologically distinct from extrapersonal, far space, which is less relevant for body-environment interactions (Cléry, Guipponi, Wardak, \& Ben Hamed, 2015 for review). Accordingly, research demonstrates that affordances are more likely to activate motor programs for objects located within peripersonal space than extrapersonal space (Cardellicchio et al., 2011; Costantini, Ambrosini, Tieri, Sinigaglia, \& Committeri, 2010; De Stefani et al., 2014; Kalénine, Wamain, Decroix, \& Coello, 2016; but see Tucker \& Ellis, 2001). For example, Cardellicchio et al. (2011) provided evidence of greater grasp-related motor activation when participants viewed graspable objects in reachable compared with nonreachable space.

Near-body space, therefore, is an important region for action and interaction with inanimate objects as well as people. Given our strong desire to preserve our near-body space from strangers, and to avoid entering theirs, there is an intriguing question of how this instinctive drive influences how we process action possibilities, or affordances, in space that is "near" more than one person. Accounting for the people around us is critical to successful and socially appropriate interactions with the environment. As such, investigating how close interpersonal proximity affects action planning and related perception is fundamental to understanding how these processes operate in an ecologically valid way. The purpose of this study was to investigate how manipulable objects are processed within space that is shared between two human strangers, henceforth referred to as social space.

Although little is known of object affordances in social space, some research suggests that other action-related processes are modulated within space shared between people. 
Findings from Constable, Pratt, Gozli, and Welsh (2015) indicated that areas of a participant's near space were coded as less action-relevant when a co-actor's hands entered it. Interestingly, in this case only an active co-actor encroaching on the participant's space modulated stimulus processing; the same results did not emerge when the experimenter merely sat across from the participant, passively observing. The authors suggested that the way space is represented as "near" in egocentric terms changes when another actor enters that space due to the way the other represents it as their own near, action space. Similarly, neuroscience research into peripersonal space has shown that the boundary of what the brain codes as actionable space is flexible and dynamic (Maravita, Spence, \& Driver, 2003 for review). Findings from Teneggi, Canzoneri, Di Pellegrino, and Serino (2013) suggest that the boundary of actionability can be socially modulated. They showed that another person standing nearby caused an inward contraction of peripersonal space boundaries. When the other person demonstrated intent to cooperate, however, peripersonal space boundaries expanded to include the co-actor. This research suggests that coding of shared, social space involves a complex and interdependent representation of one's own near-body space and that of other actors.

In light of the research reviewed earlier, and given our strong desire to maintain comfortable social distances whenever possible, we propose that there is a perceptual division of space between two strangers acting independently in close proximity. This division may modulate the processing of action-relevant stimuli within social space, and we reasoned that this modulation would apply to object affordances. If attending to nearby objects activates affordance-related motor programs (Cardellicchio et al., 2011; Costantini et al., 2010; De Stefani et al., 2014; Kalénine et al., 2016), and if close space between two strangers is segmented or divided to preserve independent near-body space boundaries, then an object in shared, social space is likely to be processed differently than if it were located near one person only. The present study sought to answer the following question: If an object located in near space is closer to another actor, does it still activate affordance-related motor codes?

\section{Experiment 1}

To investigate object affordances in social space, participants in Experiment 1 performed an object recognition task both alone and together with a stranger (confederate) standing in close proximity. Both the participant and the confederate viewed object images from opposite sides of a flat screen (Fig. 1). Objects either appeared nearer the participant or nearer the confederate but, importantly, were always within the participant's close, reachable space.
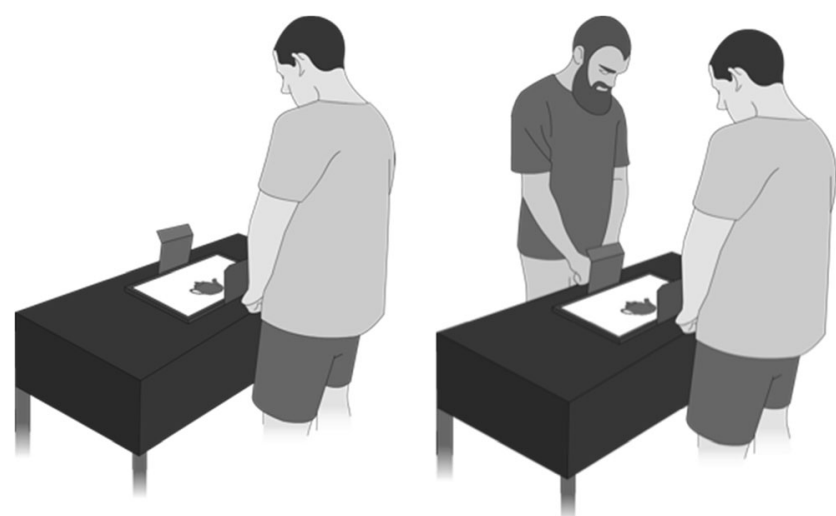

Fig. 1 Experimental set-up when participants performed the task alone (left panel) and with the confederate (right panel)

To test affordance-related motor processing, the study employed Tucker and Ellis' (1998) object inversion task. Participants viewed object images and responded with a bimanual key press to the objects' upright or inverted orientation. The object inversion task lends itself well to the current paradigm, which required two actors to view the images from opposite sides (Fig. 1). That is, two people facing one another can perform the task concurrently, despite their contrasting viewpoints, because an object appearing upright for one person appeared inverted to the other. Critically, in this task all objects afforded a single-handed grasp and appeared with handles facing the left or right. Although the stimulus feature of handle orientation is task-irrelevant, this task elicits a robust response speed advantage for trials in which object handle (left, right) and response hand (left, right) correspond rather than conflict (Iani, Baroni, Pellicano, \& Nicoletti, 2011; Pappas, 2014; Riggio et al., 2008; Saccone, Churches, \& Nicholls, 2016; Tucker \& Ellis, 1998). This congruency effect is primarily thought to reflect a match between the action afforded by the object (left- or right-handed grasp) and the action performed in making a response (left- or right-handed key press). ${ }^{1}$

The following results were predicted. The typical handle congruency effect was expected to emerge in response times (RTs). That is, faster responses were expected for trials in which object handles (left, right) matched rather than conflicted with response hand (left, right). The critical prediction was that the handle effect would interact with alone/joint condition and object proximity (near participant, far from participant). It was reasoned that if social space is divided or segmented between strangers to preserve near-body space boundaries, then in the presence of the confederate, only objects closer to the participant would elicit the handle effect. When

\footnotetext{
${ }^{1}$ There is evidence that a spatial compatibility mechanism also underlies this handle congruency effect (Cho \& Proctor, 2010, 2011; Lien, Gray, Jardin, \& Proctor, 2014); however, it is likely that this mechanism contributes to the effect, rather than explaining it entirely (Pappas, 2014; Saccone et al., 2016; Symes, Ellis, \& Tucker, 2005).
} 
participants performed the task alone, however, we expected the effect to emerge for objects in both locations because both objects were in reachable space.

\section{Method}

Participants We conducted a priori power analyses to inform participant recruitment numbers (G*Power; Faul, Erdfelder, Lang, \& Buchner, 2007). For $\alpha$ of 0.05 and a power $(1-\beta)$ of 0.95 , it was determined that 33-42 participants were required to detect an interaction of moderate effect size $\left(\eta^{2}{ }_{p}=\right.$ $0.25-0.30$ ). Thus, we recruited approximately 40 participants, which is highly consistent with previous studies employing joint task paradigms (Constable et al., 2015; Sebanz, Knoblich, \& Prinz, 2003). Thirty-nine right-handed ( $f=20$, $\mathrm{m}=19$; mean age 24.51 years) Flinders University students participated for a small reimbursement. Handedness was measured by the Flinders Handedness Inventory (Nicholls, Thomas, Loetscher, \& Grimshaw, 2013). Participants were English speakers who reported normal or corrected-tonormal vision and gave written, informed consent to participate. The study was approved by the Flinders University Social and Behavioural Research Ethics Committee.

Apparatus Stimuli were presented with a Dell Optiplex 745 PC and a LCD 23" monitor (5,182 $\mathrm{mm}$ x 2,921 mm) using Eprime 2.0 software (Psychology Software Tools, Inc.; www. pstnet.com/E-prime/e-prime.htm). The monitor was embedded in a table, facing upwards, $790 \mathrm{~mm}$ from the floor (Fig. 1). The table was 1,200-mm long and 600-mm wide. A sufficiently narrow table was necessary for two reasons: a) to ensure the actors were in close proximity, encroaching on one another's personal space (Kennedy, Gläscher, Tyszka, \& Adolphs, 2009; Lloyd, 2009; Szpak, Nicholls, Thomas, Laham, \& Loetscher, 2016; Tajadura-Jiménez, Pantelidou, Rebacz, Västfjäll, \& Tsakiris, 2011), and b) to confirm object stimuli were in the reachable, peripersonal space of both actors (Cardellicchio et al., 2011; Costantini et al., 2010; De Stefani et al., 2014). Responses were recorded via a numeric keypad, located at the participant's mid-sagittal plane, in line with the screen's horizontal centre. The confederate responded via an identical keypad but his responses were not recorded. The keypads were placed within black cardboard boxes to obscure responses from the other's view. We wanted the actors to focus on the experimental display, rather than attending to one another's responses. The experimenter monitored the session using a small closed-circuit video camera.

Stimuli Stimuli were colour photographs of 30 highly graspable objects (e.g., kettle, mug, watering can) that had an objectively correct upright orientation during use. A single, colour photograph of each object was obtained from Shutterstock's online database. All objects afforded a single- handed grasp and appeared with handles facing left or right. Object location was varied distally with respect to the participant, with objects' inner edges $20 \mathrm{~mm}$ from centre. Because of the narrow table, all objects were in the participant's reachable space (within a range of approximately $150-450 \mathrm{~mm}$ ) but were relatively near to or far from the participant. Each object appeared in two horizontal orientations (handle facing participant's left, right), two vertical orientations (upright, inverted), and two locations (near participant, far from participant), resulting in 240 unique trials. Objects ranged in size to be in approximate proportion to one another; for example, the hand saw $(210 \mathrm{~mm} \times 72 \mathrm{~mm})$ was considerably larger than the vegetable peeler $(100 \mathrm{~mm} \times 17 \mathrm{~mm})$.

Procedure Participants entered the laboratory to find the confederate (male, aged 21 years) already present. The confederate was described as "the other participant." He was instructed to maintain a neutral demeanour throughout the session and not to initiate any conversation or to engage greatly with the participant. The experimenter explained to the participant and confederate (henceforth referred to as the actors) that the experiment comprised three blocks, one each that they would perform alone and one together, although in reality only the two blocks, including the participant, were performed. The actors then gave informed consent to participate.

The experiment began with 12 practice trials, which the actors completed together, followed by two experimental blocks, each comprising 240 trials ( 30 objects $\times 2$ horizontal orientations $\mathrm{x} 2$ vertical orientations $\mathrm{x} 2$ locations). Thus, each object appeared eight times in each block. In the context of other studies employing the object inversion task, this degree of stimulus repetition is comparable to Tucker and Ellis (1998) and far less than Iani et al. (2011), who repeated each stimulus 80 times. Blocks varied by alone/joint condition (alone, with confederate), the order of which was counterbalanced across participants. The Flinders Handedness Inventory was completed in between blocks, when participants also were offered a short break.

The actors were asked to respond to each object's "normal" or "inverted" appearance as quickly and accurately as possible, regardless of object location. Responses were left and right key presses, with response mapping counterbalanced across participants. The actors stood during the task, primarily because there was not sufficient room under the narrow table to accommodate a seating position for both actors during the joint block. Actors were asked to stand with their thighs touching the table so that their viewing position remained constant throughout the experiment. Stimulus presentation order was randomised.

In the joint block, participant and confederate responded to each stimulus concurrently, giving opposing correct responses given their contrasting viewpoints. Only the participant's responses were recorded, however. Because of the width of the 
table, when performing the task together the actors stood approximately $600-\mathrm{mm}$ apart, with a distance of approximately $450 \mathrm{~mm}$ between their hands. The confederate was quiet and focused on the task, paying little attention to the real participant, and maintained a constant gaze on the experimental display to encourage the participant to behave likewise.

Each trial began with a central fixation cross $(18 \mathrm{~mm} \times 18$ $\mathrm{mm}$ ), shown for $500 \mathrm{~ms}$, followed by the stimulus, which remained on screen for $1,500 \mathrm{~ms}$ or until the participant responded. If no response was made (missed trials), onscreen text feedback reminded the actors to respond as quickly as possible. This message, displayed for 2,000 ms, appeared in two orientations so it could be read by both participant and confederate. Where participants responded in time, the intertrial interval was $500 \mathrm{~ms}$. This trial procedure is consistent with previous versions of the object inversion task (Iani et al., 2011; Pappas, 2014; Riggio et al., 2008; Saccone et al., 2016; Tucker \& Ellis, 1998), including the use of fixed inter-trial intervals and the lack of catch trials.

After the two experimental blocks were completed, participants were told that there would not be a third block and that the experiment was finished. Participants were debriefed appropriately and thanked for their time.

\section{Results and discussion}

Data analysis For simplicity, and because we were interested in spatial congruency effects relating to object handle and response, data were collapsed across response (left, right) and object handle side (left, right) to create the factor of handle (congruent, incongruent). Congruent trials were those in which the response and handle side matched, and incongruent trials were those in which they conflicted. Although this congruency effect reliably manifests in response speed, error rates often fail to produce an effect (Constable, Kritikos, \& Bayliss, 2011; Iani et al., 2011; Riggio et al., 2008; Saccone et al., 2016). Furthermore, research suggests that affordance processes are not the primary mechanism underlying the effect in errors when it is found (Saccone et al., 2016). Accordingly, only the RT data are reported in the current manuscript. Analyses with respect to error rates are available in the supplementary material. The key interaction, representing the study's experimental hypothesis, was not significant.

Although the factors of participant sex and stimulus upright/inverted orientation did not inform the study's a priori hypothesis, it was considered they could influence responses. In light of the social nature of the paradigm and given that only a male confederate was used, it is possible that participant sex in particular could be of interest to some readers. However, including these additional variables would result in a highly complex factorial design and a 5-factor ANOVA model that would be difficult to interpret. Accordingly, these additional factors are not included in the analysis presented below, but results from the larger, 5-factor ANOVA model are available in the supplementary material. Of note, there was no main effect of participant sex and neither sex nor upright/ inverted orientation interacted with alone/joint condition and therefore do not counteract the study's key hypothesis. All significant effects detailed below remained significant in the larger, 5-factor ANOVA model.

Data from two male participants were excluded from all analyses due to error rates above chance, which also were above three standard deviations from the sample mean $(16.350 \%, S D=18.534)$. Once these participants were removed, the group error rate was $12.490 \%(S D=8.025)$.

RTs A 2 (alone/joint condition; alone, confederate present) x 2 (handle; congruent, incongruent) x 2 (object proximity; near participant, far from participant) repeated measures ANOVA was conducted on mean RTs from correct trials. There were main effects of alone/joint condition and handle (Fig. 2). Participants made faster responses in the presence of the confederate $(M=563.193, S D=72.335)$ than when performing the task alone $(M=603.196, S D=75.248), F(1,36)=11.828$, $p=0.001, \eta^{2}{ }_{p}=0.247$. This likely reflects a social facilitation effect (Zajonc, 1965), whereby performance is enhanced in the presence of others. Mean RTs also showed the typical handle effect (Iani et al., 2011; Pappas, 2014; Riggio et al., 2008; Saccone et al., 2016; Tucker \& Ellis, 1998): participants were faster to respond with the hand that was congruent with the object's handle $(M=576.660, S D=67.669)$ rather than incongruent $(M=598.730, S D=63.265), F(1,36)=16.647, p$ $<0.001, \eta^{2}{ }_{p}=0.316$. There was no main effect of object proximity, $F(1,36)=0.684, p=0.414, \eta_{p}^{2}=0.019$ and no significant 2-way interactions, all $F_{\mathrm{s}}<0.945, p \mathrm{~s}>0.337$, but there was a significant 3-way interaction between alone/joint condition, handle, and object proximity, ${ }^{2} F(1,36)=4.451, p=$ $0.042, \eta_{p}^{2}=0.110$.

To explore the significant 3-way interaction, separate 2 (handle) x 2 (object proximity) repeated measures ANOVAs were performed for the alone/joint conditions. There was no significant interaction between handle and object proximity for the alone condition, $F(1,36)=0.649, p=0.426, \eta^{2}{ }_{p}=$ 0.018 , indicating a comparable handle effect for both near (congruent $M=597.141, S D=74.244$; incongruent $M=$ 608.765, $S D=76.440$ ) and far objects (congruent $M=$ 594.702, $S D=83.864$; incongruent $M=612.177, S D=$ 77.301). There was, however, a significant interaction between handle and object proximity for the confederate condition, $F(1,36)=6.554, p=0.015, \eta_{p}^{2}=0.154$. Paired sample ttests revealed that, when the confederate was present, the handle effect was elicited by near objects (congruent $M=$

\footnotetext{
${ }^{2}$ Of note, this interaction was also significant in the larger, 5-factor ANOVA detailed in the supplementary material, $F(1,34)=4.957, p=0.033, \eta^{2}{ }_{p}=$ 0.127 .
} 


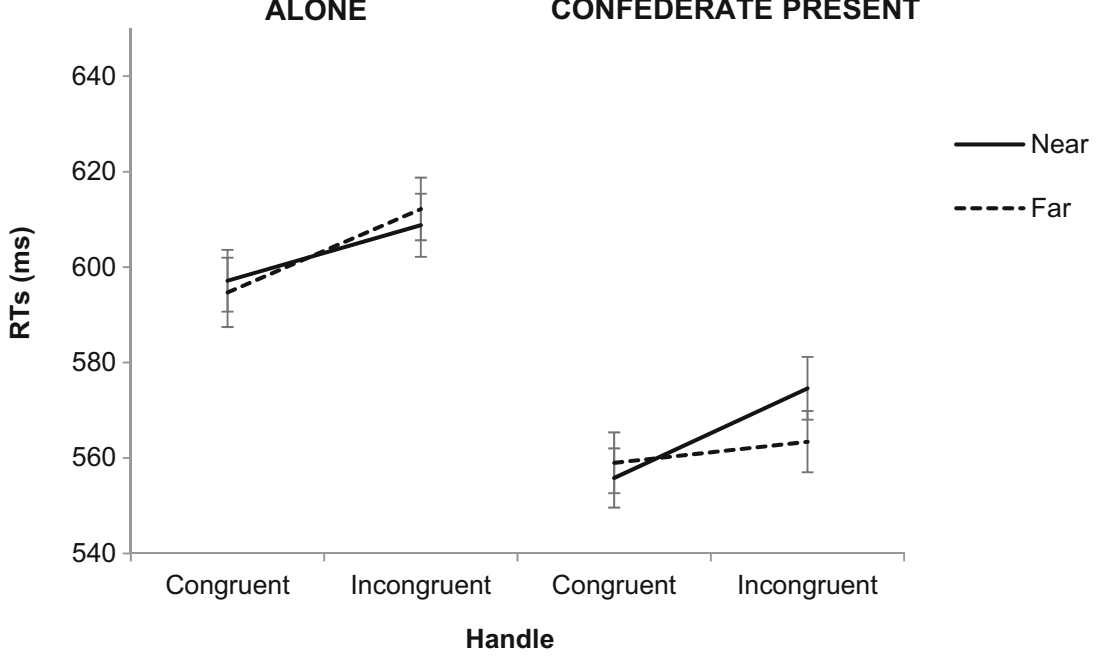

Fig. 2 Mean correct RTs (ms) across handle and proximity factors for the alone condition (left) and the confederate condition (right) in Experiment 1. Error bars represent a standard error of the mean calculated within-subjects for each condition

555.806, $S D=75.942$; incongruent $M=574.579, S D=$ $74.105), t(36)=3.731, p=0.001, d=0.614$, but not far objects (congruent $M=558.989, S D=77.818$; incongruent $M=$ 563.399, $S D=68.257), t(36)=1.095, p=0.281$.

Given the importance of the above contrasts to the study's hypothesis, confirmatory Bayesian statistics were conducted. These analyses produced Bayes factors, which can be interpreted as odds ratios, quantifying the likelihood of the data under one hypothesis compared to another (Wetzels et al., 2011). Bayesian ANOVAs were conducted in JASP (JASP Team, 2017) using default priors. For the alone condition, the model without the handle*object proximity interaction term was preferred to the model including it, with a Bayes factor of 3.340. This finding confirms that the handle effect was comparable for near and far objects when participants were alone. Conversely, for the confederate condition, the model that included the handle*object proximity interaction was favoured by a Bayes factor of 3.141. This finding supports a significant difference in the handle effect for near and far objects in the confederate condition. Thus, Bayesian analyses accord with the results described above.

These findings support the study's central hypothesis. All objects were located within near-body, reachable space of participants, but when a stranger was acting in close proximity, only nearer objects elicited the handle effect. When participants were alone, however, the effect was evident for both near and far objects. In line with evidence for modulated processing of action-relevant stimuli in social space (Constable et al., 2015; Teneggi et al., 2013), and given that humans are instinctively motivated to preserve personal space boundaries (Evans \& Wener, 2007; Hirsch \& Thompson, 2011; Soper \& Karasik, 1977), in Experiment 1 it seems that another actor in close proximity led to a perceptual division of social space, such that far objects no longer produced the same degree of lateralised, affordance-related motor activation.

One consideration is that responses made by the confederate may have influenced participants' performance in the joint condition. In particular, participant responses could have been slowed in instances where the confederate gave a response that did not match the participant's (Tsai, Kuo, Jing, Hung, \& Tzeng, 2006). We feel that this is unlikely to be a concern for the following reasons. First, the actors' hands were occluded and so the visible movement cues as to the response given were slight at most. Second, the experimental procedure was such that trials moved quickly from one to the next to ensure that the actors' gaze and attentional focus were maintained on the display. Furthermore, the experimenter monitored the session from outside the testing room to ensure the actors were performing the task in this manner. Last, due to the actors' opposing viewpoints, a leftward object handle from the participant's perspective was in fact a rightward handle from the confederate's (Fig. 1). Given that the actors gave opposing correct responses (i.e., upright vs. inverted) and these responses were made with opposing response hands (e.g., left for upright, right for inverted), a correct, handle-congruent or incongruent response for one actor was in fact likewise for the other. Thus, the confederate's responses were unlikely to have slowed participant responses or represented a confound in the joint condition.

We have interpreted the current findings as a social phenomenon, but alternatively it is possible that a nonsocial joint condition would produce the same pattern of results. Perhaps the human, biological agency of the confederate was not critical in eliciting these effects. The confederate may simply have served as a distractor that participants attempted to ignore, leading them to 
neglect near-confederate space and stimuli within it (Tipper, 1985). Certainly, it is possible that the close proximity of the confederate caused an inward or proximal withdrawal of the participant's attention (Szpak et al., 2015), although such a process would not necessarily preclude the proposed mechanism of a perceptual division of social space. Critically, in Experiment 1, social presence differentially influenced RTs only with respect to object affordance, with no overall reduced RT for far objects. In other words, if a simple distractor mechanism explained the current results, one would expect slower responses overall for far objects in the joint condition, which was not the case. Even if there was an element of attentional withdrawal or an attentional mechanism, it only affected the visuomotor processing of stimuli (i.e., motor congruency between responses and objects' affordances), not object identification overall. As such, the findings speak against a general attentional or distractor mechanism.

Although the data are not consistent with a simple distractor explanation, it is important to provide stronger evidence that these findings represent a social phenomenon. To confirm a social mechanism underlying Experiment 1's key interaction, two further experiments were conducted, each employing a nonhuman distractor object in the joint condition instead of a human confederate. We adapted a methodology from Dolk, Hommel, Prinz, and Liepelt (2013), as described below, whereby the social nature of the distractor objects was reduced in a stepwise manner across the experiments. As we were distinguishing between a social rather than general, attentional mechanism, highly visually and/or aurally salient distractor objects were chosen for the joint conditions in the following two experiments.

\section{Experiment 2}

Experiment 2 was conducted to determine whether Experiment 1's key finding was truly a social effect, dependent on the human, biological agency of the confederate. The joint condition in Experiment 2 was adapted from the spatial compatibility literature, in which a similar issue has arisen. To explain, Sebanz et al. (2003) conducted a collaborative task between participant pairs, whereby each member was asked to respond to one of two stimulus colours. They found that participants sitting on the left were faster to respond to left- rather than right-located stimuli and vice versa for participants sitting rightward. This stimulus-response spatial compatibility disappeared when participants performed the task alone, even though participants were still sitting in left or right locations. Dolk et al. (2013) subsequently tested whether this joint task effect was truly social in nature with a series of experiments employing visually and/or aurally salient, nonhuman objects in the joint condition. ${ }^{3}$ The authors systematically reduced the "socialness" of the objects across each of their experiments. The first, most social of these nonhuman items was a gold, Japanese waving cat statue (Fig. 3).

Thus, Experiment 2 employed the method from Experiment 1, but in the joint condition participants performed the task in the presence of a small, gold, waving cat statue rather than a human stranger. In line with Dolk et al.'s (2013) reasoning, the statue was a sufficiently visually and aurally salient nonhuman object due to its moving arm.

The following predictions were made. If the finding from Experiment 1 was driven by a general attentional, distractor mechanism, then similar results were expected in Experiment 2. Specifically, the critical 3-way interaction from Experiment 1 would be replicated, whereby the joint (cat) condition elicited a handle effect for near but not far objects, whereas the alone condition produced the effect for objects in both locations. If the key finding from Experiment 1 did in fact represent a social phenomenon, reflecting visuomotor modulation within social space, then Experiment 2 was not expected to produce a 3-way interaction between alone/joint condition, handle, and object proximity. In this case, it was expected that objects in both locations would elicit handle effects, regardless of alone/joint condition.

\section{Method}

Participants Forty-one, right-handed (20 females, mean age 21.98 years) Flinders University students participated for a small reimbursement. Handedness was measured by the Flinders Handedness Inventory (Nicholls et al., 2013). Participants were English speakers who reported normal or corrected-to-normal vision and gave written, informed consent to participate. The study was approved by the Flinders University Social and Behavioural Research Ethics Committee.

Apparatus and stimuli All apparatus and stimuli were identical to Experiment 1 with the addition of the cat statue (Fig. 3). The cat measured 205-mm high, $120-\mathrm{mm}$ wide, and 110$\mathrm{mm}$ deep. Its left arm waved at a frequency of $0.55 \mathrm{~Hz}$, accompanied by a soft, rhythmic noise.

Procedure Procedures were the same as Experiment 1 except with respect to the confederate. Rather than performing the

\footnotetext{
${ }^{3}$ To clarify, although we have adapted Dolk et al.'s (2013) methodology to investigate a social mechanism, we note the different applications of this methodology across their study and ours. Dolk et al. employed nonhuman objects to investigate a referential, event coding account of the joint task effect in their study, rather than the attentional, distractor account we have proposed.
} 


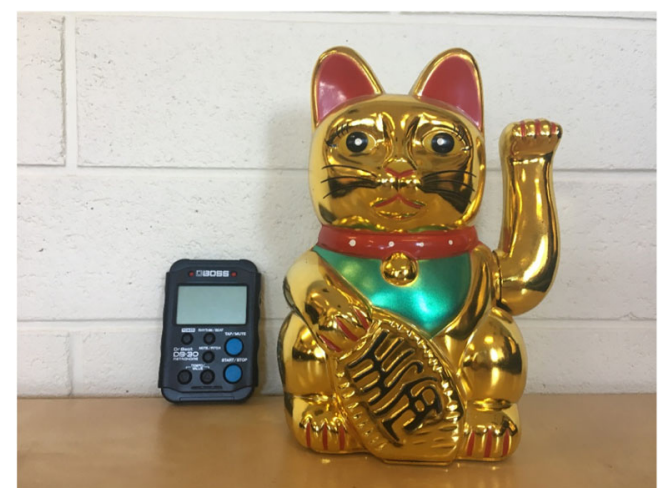

Fig. 3 The cat statue and digital metronome employed in the joint conditions of Experiments 2 and 3, respectively

task with another person, participants were asked to complete two experimental blocks: one in the presence of the cat statue. During the joint condition, the cat was positioned on the table in place of the confederate's key pad to ensure it was sufficiently visually salient.

\section{Results and discussion}

Data analysis The factor of participant sex was not included in the following analyses. Although some evidence points to sex differences in cognitive processing (Stoet, 2010), Experiment 1 did not reveal any effects in relation to participant sex and so it was considered unlikely to be a variable of interest in Experiment 2. This reasoning notwithstanding, preliminary analyses performed on RT data from Experiment 2 revealed neither a main effect nor any interactions with the factor of participant sex.

Data from two male participants were excluded from analyses due to error rates greater than three standard deviations from the group mean $(10.520 \%, S D=7.896)$. After removing these two participants, the group error rate was $9.220 \%(S D=$ 5.492). Analyses of error rates are presented in the supplementary material. The study's critical 3-way interaction was not significant.

RTs A 2 (alone/joint condition; alone, cat present) x 2 (handle; congruent, incongruent) x 2 (object proximity; near participant, far from participant) repeated measures ANOVA was conducted on mean RTs from correct trials (Fig. 4). Unlike Experiment 1, there was no main effect of alone/joint condition (alone $M=609.806, S D=62.927$; joint $M=605.635, S D$ $=79.310), F(1,38)=0.106, p=0.747, \eta_{p}^{2}=0.003$, suggesting the effect from Experiment 1 was in fact social facilitation. There was a significant main effect of handle, $F(1,38)=$ $11.225, p=0.002, \eta_{p}^{2}=0.228$, reflecting the typical response speed advantage for congruent $(M=601.798, S D=62.995)$ over incongruent handles $(M=613.642, S D=57.643$; Fig. 4). The main effect of object proximity was not significant, $F(1$,
38) $=1.037, p=0.315, \eta_{p}^{2}=0.027$, and there were no significant 2-way interactions, all $F \mathrm{~s}<0.019, p \mathrm{~s}>0.890$.

The critical 3-way interaction between alone/joint condition, handle and object proximity was not significant, $F(1,38)$ $=3.613, p=0.065, \eta_{p}^{2}=0.087$, but it was close to the conventional cutoff for statistical significance. As shown in Fig. 4, there appears to be a trend for a weaker handle effect for far compared with near objects in the cat present condition, which is consistent with Experiment 1. In light of this pattern, and given its theoretical importance to the study, this nonsignificant 3-way interaction was investigated further. Separate 2 (handle) x 2 (object proximity) repeated measures ANOVAs were performed for the alone/joint conditions. The interaction between handle and object proximity was neither significant in the alone, $F(1,38)=1.981, p=0.167, \eta_{p}^{2}=0.050$, nor cat present conditions, $F(1,38)=2.059, p=0.159, \eta^{2}{ }_{p}=0.051$.

Bayesian ANOVAs were once again conducted for these contrasts. The model excluding the handle*object proximity interaction term was preferred for both the alone (Bayes factor $=1.917$ ) and joint conditions (Bayes factor $=2.119$ ). These results provide further evidence for statistically comparable handle effects for near and far objects in both the alone and cat present conditions, consistent with analyses described above.

Last, it was considered the visual asymmetry of the cat's waving left arm might represent a confound and influence responses in the cat present condition. It was particularly important to establish that this asymmetry did not affect responses because in Experiment 1 the participants' hands were occluded. Accordingly, the original 2 (alone/joint condition) $\mathrm{x}$ 2 (handle) x 2 (object proximity) repeated measures ANOVA model was performed again but with the factor of handle (congruent, incongruent) replaced with response hand (left, right) and object orientation (handle facing left, right). Results from this larger, 4-factor model are available in the supplementary material. Importantly, the critical 4-way interaction between alone/joint condition, object proximity, response hand and object orientation remained nonsignificant, $F(1,38)=3.495, p=0.069, \eta^{2}{ }_{p}=0.084$, and alone/joint condition did not interact with any other factors, $F \mathrm{~s}<1.891$, $p \mathrm{~s}>0.177$. These results strongly suggest that the cat's waving arm did not asymmetrically bias participants' attention during the cat present condition.

Overall, these findings support a social interpretation of Experiment 1's data. Experiment 2 did not replicate the key finding from Experiment 1, suggesting the presence of a human is necessary to elicit the results. The data, however, are not as convincing as expected. The cat present condition produced a similar pattern of data to that elicited by the human confederate in Experiment 1, although this effect was not statistically significant. Bayesian analyses likewise pointed against any meaningful differences across alone/joint conditions. Given that human-like features and movement can lead 


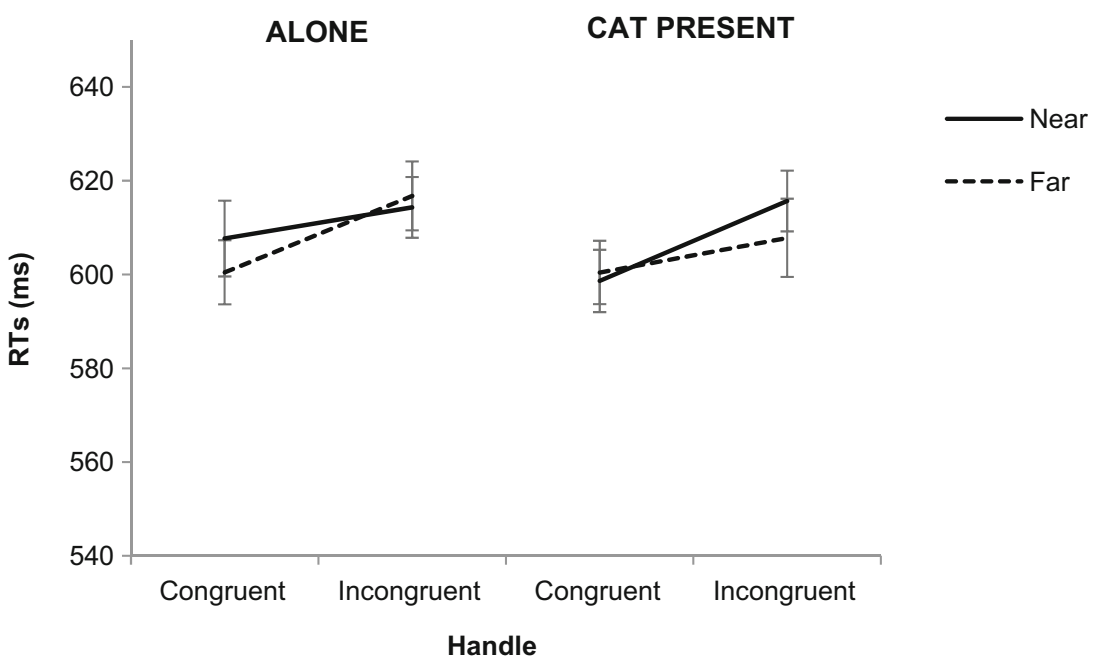

Fig. 4 Mean correct RTs (ms) across handle and proximity factors for the alone condition (left) and the cat condition (right) in Experiment 2. Error bars represent a standard error of the mean calculated within-subjects for each condition

to anthropomorphising of mechanical devices (Prakash \& Rogers, 2015), we suggest that the anthropomorphic features of the cat statue (i.e., face, moving arm) may have introduced a social context to Experiment 2 to a degree, but not strongly enough to produce a statistically significant effect. This suggestion is in line with Dolk et al.'s (2013) reasoning regarding the element of socialness association with the cat. Moreover, as in Experiment 1, on-screen instructions appeared in two orientations, facing both toward and away from the participant. Having instructions directed to the cat statue may have encouraged its anthropomorphisation.

On balance, Experiment 2 provides support for a social account of the data from Experiment 1. More convincing evidence is required, however, to draw stronger conclusions about the mechanism underlying the study's key finding. Accordingly, Experiment 3 included a joint condition employing a distractor object with no human-like features at all.

\section{Experiment 3}

Experiment 3 determined whether a salient but inanimate object with equivocally no social features would produce results similar to Experiment 1. Once again, based on methodology from Dolk et al. (2013), a digital metronome was chosen for the joint condition in Experiment 3 (Fig. 3). A metronome is completely devoid of human-like features, does not move, but is a highly salient object due to its auditory output.

In terms of predicted results, if Experiment 1's key finding is explained by a general attentional, distractor mechanism, then Experiment 3 was expected to reproduce the critical 3way interaction from Experiment 1. Specifically, in the joint (metronome) condition, near but not far objects would elicit the handle effect whereas objects in both locations would produce the effect in the alone condition. If Experiment 1's key finding is truly a social phenomenon, then Experiment 3 was expected to elicit a comparable handle effect regardless of alone/joint conditions and object proximity, with no significant a 3-way interaction.

\section{Method}

Participants Thirty-six right-handed (32 females, mean age 21.69 years) Flinders University students participated for a small reimbursement. Handedness was measured by the Flinders Handedness Inventory (Nicholls et al., 2013). Participants were English speakers who reported normal or corrected-to-normal vision and gave written, informed consent to participate. The study was approved by the Flinders University Social and Behavioural Research Ethics Committee.

Apparatus, stimuli and procedure All apparatus, stimuli, and procedures were identical to Experiment 2 except for the inclusion of a small, black, digital BOSS DB-30 metronome (Fig. 3) in place of the cat statue. Lying flat, the metronome measured 15-mm high, 60-mm wide, and 88-mm long. The metronome was placed face-down on the table such that its visual display was hidden and it served primarily as an auditory distractor. It beeped audibly at a rate of 80 beats per minute (Dolk et al., 2013).

\section{Results and discussion}

Data analysis Error rates from every participant fell within three standard deviations from the group mean $(12.008 \%, S D$ $=5.423$ ), and so all participants' data were analysed. Analyses of error rates are presented in the supplementary material. The study's critical 3-way interaction was not significant. 


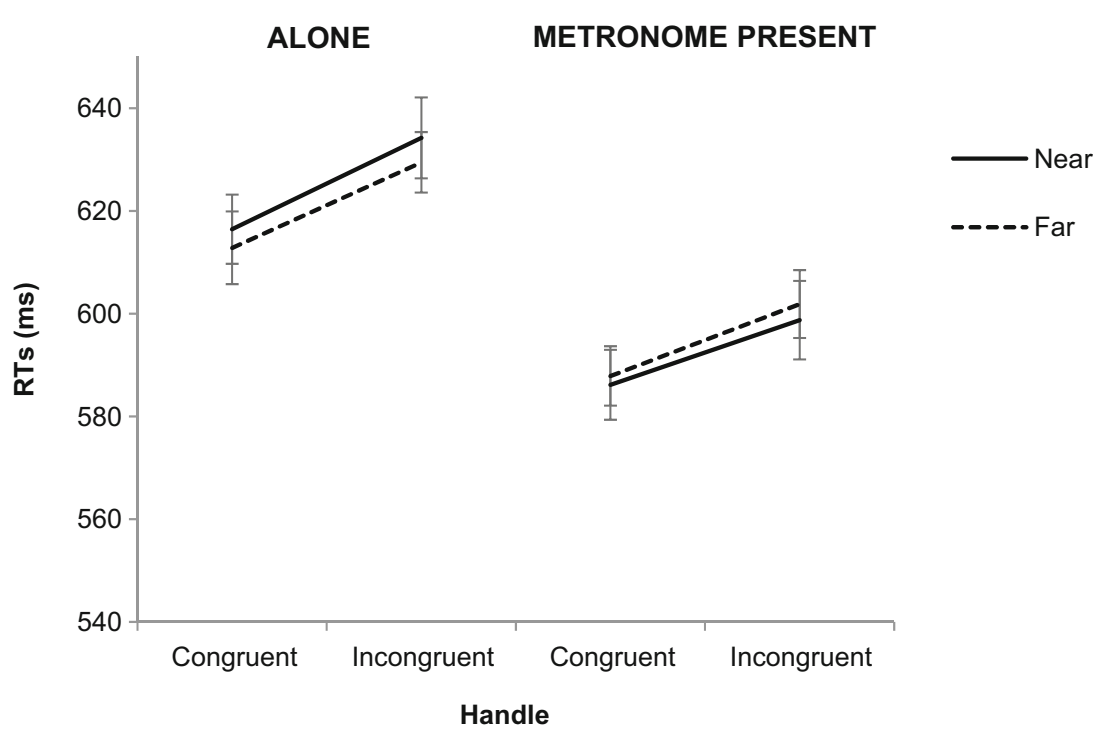

Fig. 5 Mean correct RTs (ms) across handle and proximity factors for the alone condition (left panel) and the metronome condition (right panel) in Experiment 3. Error bars represent a standard error of the mean calculated within-subjects for each condition

RTs A 2 (alone/joint condition; alone, metronome present) $\mathrm{x} 2$ (handle; congruent, incongruent) x 2 (object proximity; near participant, far from participant) repeated measures ANOVA was conducted on mean RTs from correct trials. There was a significant main effect of alone/joint condition, $F(1,35)=$ $6.497, p=0.015, \eta_{p}^{2}=0.157$, reflecting faster responses when the metronome was present $(M=593.676, S D=81.078)$ than absent $(M=623.253, S D=94.151$; Fig. 5). The reason for this finding is unclear, because it contrasts both Dolk et al. (2013), who found slower responses in their metronome condition, and also the current Experiment 2, in which there was no difference in RTs across alone and cat present conditions. It is possible that the metronome had an especially altering or arousing effect, which can lead to faster RTs, particularly in the case of loud auditory stimuli (Ulrich, 1996). This explanation is consistent with arousal or alertness accounts of social facilitation effects (Strauss, 2002; Zajonc, 1965), such as that observed in our Experiment 1, and also in Constable et al. (2015), who found the greatest degree of social facilitation in the active co-actor condition, and the least in the passive, indirect observer condition. There also was a significant main effect of handle, $F(1,35)=11.457, p=0.002, \eta^{2}{ }_{p}=0.247$, with the typical response speed advantage for congruent $(M=$ 600.676, $S D=80.319)$ over incongruent handles $(M=$ $616.092, S D=83.238)$. This finding is consistent with both Experiments 1 and 2 . The main effect of proximity was not significant, $F(1,35)=0.181, p=0.673, \eta_{p}^{2}=0.005$, and there were no significant 2-way interactions, all $F \mathrm{~s}<1.879, p \mathrm{~s}>$ 0.179 . Most importantly, the 3 -way interaction between alone/joint condition, handle and object proximity was not significant, $F(1,35)=0.115, p=0.737, \eta_{p}^{2}=0.003$.

For consistency across experiments, Bayesian analyses were again performed. The model excluding the handle*object proximity interaction term was preferred for both the alone (Bayes factor $=4.150)$ and joint conditions $($ Bayes factor $=$ 4.456). These results confirm the comparable handle effects for near and far objects in both the alone and metronome conditions in Experiment 3.

Overall Experiment 3's results support a social interpretation of Experiment 1's key finding. The findings suggest that an entirely nonsocial, aurally salient distractor object does not differentially affect affordance-related visuomotor processing of near and far objects. These findings also indicate that the somewhat ambiguous results from Experiment 2 are most likely attributed to the anthropomorphic features of the cat statue. Although the cat also differed from the metronome in that it was visually as well as aurally salient, the main effect of alone/joint condition in Experiment 3 demonstrates that the metronome was a highly salient distractor object, which captured participants' attention but had no impact on the handle effect.

One final consideration with respect to the results produced in the study is that differences in overall response speed could account for the divergent findings across experiments. To investigate this possibility, we examined the temporal development of the handle effect in all three joint conditions. We employed Symes, Ellis, and Tucker's (2005) method, in which participant mean quartile RTs were calculated for congruent and incongruent handle trials (Fig. 6). Critically, the overall time course of responses is highly consistent across all three joint conditions, demonstrating that global differences in response speed do not account for the divergent findings across experiments. Of further note, the time course of the handle effect is remarkably consistent for the joint conditions of Experiments 2 and 3, whereas it appears weaker in Experiment 1. This weaker overall effect in Experiment 1 


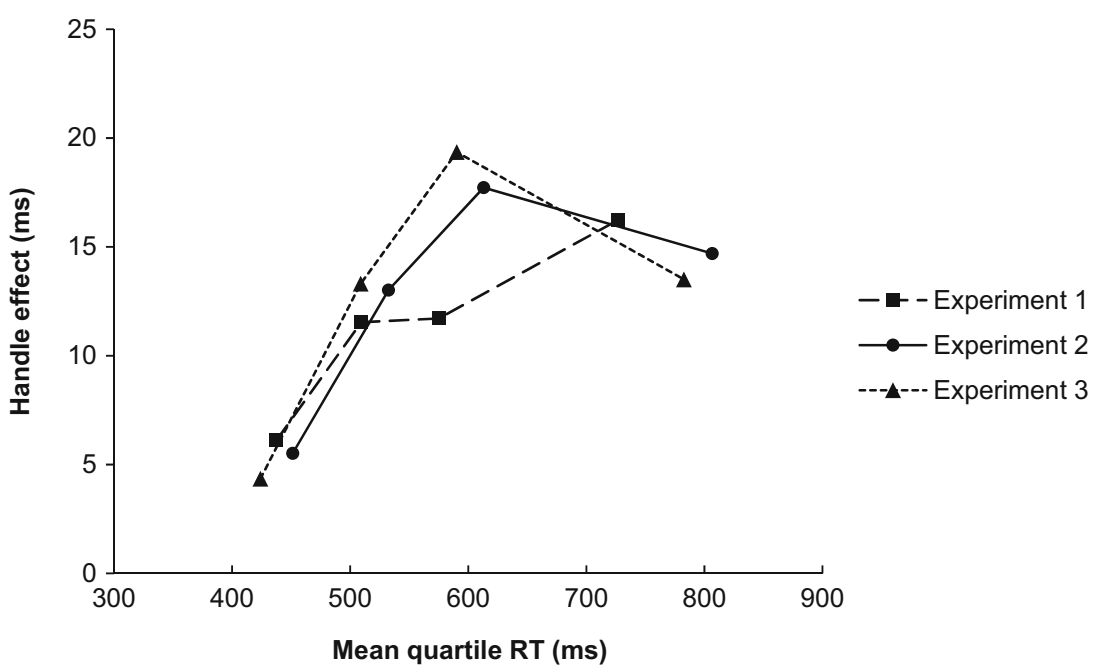

Fig. 6 Mean quartile RTs (ms) for the handle effect (incongruent - congruent) for the joint conditions of Experiments 1, 2, and 3

reflects the statistically significant difference between near and far objects and, in particular, the absent handle effect for far objects when the confederate was present.

\section{General discussion}

The present research determined how close interpersonal proximity influences visuomotor processing of manipulable objects in social space between two strangers. As the first study to investigate Tucker and Ellis' (1998) handle congruency effect in a paradigm with two human co-actors, these findings are an important extension of previous research employing single participant designs. For most humans, social encounters occur frequently throughout each day and accounting for the people around us is critical to successful and socially appropriate interactions with the environment. As such, investigating how social presence modulates the perception of action possibilities is critical to understanding these processes in a real-world context. The current set of experiments has demonstrated that although nearby objects typically elicit visuomotor congruency effects, objects that are within reach but are closer to a stranger do not activate affordance-related motor codes to the same degree. These results suggest that space shared between two human strangers can be divided to preserve one another's near-body space, and in turn this process influences visuomotor coding of stimuli within that social space.

Converging evidence from the three current experiments rules out an alternative, general attentional account of Experiment 1's key finding. When participants were alone, all objects within near space evoked the handle effect, whereas in the presence of the confederate, only objects nearer the participant produced affordance-related motor activation strongly enough to elicit the effect. It was considered that the confederate may have simply served as a distractor that participants attempted to ignore, leading to reduced attentional processing of stimuli in his proximity. If this were the case, we would have expected inhibited responses overall for far stimuli in the joint condition, but instead the confederate differentially affected responses with respect to affordances only for far objects. Moreover, the decreasingly social, nonhuman, visually, and aurally salient joint conditions of Experiments 2 and 3 did not replicate the critical finding from Experiment 1.

In demonstrating that affordance-related processing can be socially modulated, these findings invite numerous interesting questions regarding the nature of social space, and how other social and action variables might affect visuomotor processes in this context. First, how might the current findings be modulated by other factors relating to interpersonal distance? There are individual differences in preferred social distance, which could affect coding of near-body space and action processes within it (de Vignemont \& Iannetti, 2015). To illustrate, Iachini, Ruggiero, Ruotolo, di Cola, and Senese (2015) showed that trait measures of anxiety and extraversion correlated with preferred social distance, as well as reachability estimates. Social comfort distances may vary across cultures, related to crowding norms and attitudes (Evans et al., 2000), or with the nature of the actors' social relationship, because friends typically engage at closer distances than strangers (Hall, 1966).

Although the precise mechanism underlying our results remains unknown, the research reviewed above could point to a powerful role of discomfort in social space coding. Indeed, de Vignemont and Iannetti (2015) argued that anxiety induced by social proximity might reflect an instinctive, adaptive motivation to protect one's body, which could induce a contraction of peripersonal, action space. This proposal is in line with findings indicating an association between trait anxiety and near-body space coding (Iachini et al., 2015; Sambo 
\& Iannetti, 2013). Szpak et al. (2015) similarly demonstrated that for two strangers in close proximity, attentional withdrawal was most pronounced for those displaying the most physiological arousal. Therefore, discomfort may have played a role in Experiment 1's findings, especially given that the confederate was instructed to maintain a neutral, rather than friendly, demeanour and to engage minimally with the participant. The current study did not obtain measures of participant state or trait anxiety or social discomfort; accordingly, we cannot provide direct evidence for this idea. It is, however, a plausible explanation in light of the research and arguments reviewed here and the proposed adaptive motivation for social distances in general. This potential mechanism provides an interesting and promising avenue for future research into social space coding and the nature of visuomotor processing within this space.

In considering social discomfort, it is likely that other social, contextual variables also would affect visuomotor processing in shared space. As mentioned, two friends might not demonstrate the same perceptual division of social space evident in the current study, because they typically have closer social comfort distances (Hall, 1966). Strangers who collaborate on a task might likewise feel more comfortable with one another than in the current paradigm. As de Vignemont and Iannetti (2015) note, coordinating action with another person requires a degree of trust, and so space between two people who perform an action together (e.g., one passing a cup of coffee to the other) could be coded very differently than for two strangers acting independently, for example. Pezzulo et al. (2013) likewise argued for a merging (rather than dividing) of actors' near-body spaces when they collaborate to achieve a common goal. Richardson et al. (2007) have in fact demonstrated that the way we act upon objects changes in an explicitly collaborative social context. Perhaps a collaborative, turn-taking paradigm or a task in which actors' responses complement one another might not elicit the same perceptual division of space that we found, especially given that these factors have produced variable effects in joint action paradigms (Jordan \& Knoblich, 2004).

Aside from considering how social, contextual factors impact social space, a further complication is that the way in which social space is coded could conversely influence actors' social interactions. That is, perceptual division of space between two actors could in fact incite collaboration between them in a natural, social context. For example, refraining from entering another's near-space to obtain an object could result in a request for the object to be handed from one person to the other. Considerable future research is required to investigate how social, contextual, and taskrelated factors influence and interact with social space coding and how these potentially highly complex interactions affect visuomotor processing and subsequent social interactions within that space.

It is likewise unknown how action-related variables influence object affordance processing in social space. Perhaps varying the motor capabilities or goals of the actors would influence visuomotor processes in this shared space (Pezzulo et al., 2013). Consistent with this idea, Witt, Sugovic, and Taylor (2012) demonstrated that our reported experience of an object is not only affected by our ability to act on it but also by the ability of others to act on it. Accordingly future research could investigate the visuomotor processing of object stimuli in social space when the co-actor's motor capabilities were somehow restricted. Furthermore, findings from Constable et al. (2015) suggested that an active-co-actor affects visuomotor processing in shared space differently than when the experimenter stands in as a passive but direct observer. The experimenter did not encroach on the participant's near-body space in the same way as the active co-actor in Constable et al.'s (2015) study. Regardless, including such a condition would have been a useful experiment in the context of the present study, and we acknowledge that not doing so represents a limitation in our work. Investigating affordance processing in space shared with a passive observer would have informed on the degree to which our findings were contingent on the actions of the confederate and the particular nature of the social mechanism underlying our results. In general, it remains unclear how established social action effects might interact with social space coding, especially in light of the proposed role of discomfort in preserving near-body space boundaries. Perhaps such an instinctive, adaptive mechanism is so powerful that it renders these other factors irrelevant. This possibility could further explain the findings of the current Experiment 2, in which the cat statue, a moving stimulus with human-like features, elicited a similar, but statistically nonsignificant, pattern of results to the confederate in Experiment 1.

It is likewise not yet clear how visuomotor modulation in shared space interacts with other mechanisms that can operate within social action contexts. Findings from some joint tasks indicate, for example, that participants not only understand the spatial perspective of a co-actor but that this perspective can manifest behaviourally. That is, participants' responses to the experimental stimuli can in fact sometimes reflect another's allocentric viewpoint, rather than their own, egocentric perspective (Böckler, Knoblich, \& Sebanz, 2011; Tversky \& Hard, 2009). This phenomenon may be particularly likely in paradigms where participant pairs collaborate while standing opposite one another (Frischen, Loach, \& Tipper, 2009) and view images in more than one orientation (Surtees, Apperly, \& Samson, 2016). Interestingly, though, Experiment 1 comprised of such a paradigm but participants' responses evidently did not reflect the confederate's spatial perspective, given that stimulus upright/inverted orientation did not interact with 
alone/joint condition (see Supplementary Material). Perhaps actor pairs standing opposite one another only take the other's perspective when acting in a collaborative task or context, as in studies by Frischen et al. (2009) and Surtees et al. (2016). Freundlieb and colleagues (Freundlieb, Kovács, \& Sebanz, 2016; Freundlieb, Sebanz, \& Kovács, 2017) have in fact demonstrated strong evidence for perspective taking when both actors are independently identifying the location of a target stimulus; however, in these experiments the confederate was sitting at a $90^{\circ}$ angle to the participant and in most cases the stimulus display was spatially congruent with the confederate's response configuration, and never with the participant's. Further research is needed to determine which conditions are necessary to elicit a perspective taking mechanism and, moreover, how this process interacts with social space coding. If discomfort does play a role in preserving independent nearbody space boundaries, then perhaps this adaptive process is sufficiently strong to prevent perspective taking. Overall, action planning and selection within social contexts, including the mapping of social space and affordances within it, likely reflects a complex and dynamic interplay of many social and action-related variables (Brincker, 2015; Pezzulo et al., 2013). The current study has highlighted the complex nature of this interaction and the considerable further research that is required to understand this phenomenon.

One last point relates to the distinction between social-and action-related representations of near-body space. We have discussed personal, social space separately from peripersonal, action space, because historically they are informed by discrete bodies of literature. Furthermore, it was important to highlight the relevance of both social and action processes to near-body space. In reality, however, it is unknown whether a functional distinction between social and action space representations exists. In terms of conceptual overlap, both representations refer to the space surrounding the body and serve to protect the body from and facilitate interaction with (animate or inanimate) stimuli in the environment and recent papers suggest the two representations may in fact be functionally distinct (de Vignemont \& Iannetti, 2015; Patané, Iachini, Farnè, \& Frassinetti, 2016). This issue is highly complex, and its resolution awaits considerable further, exciting research.

The present study provides an important step forward in understanding the ease with which we can navigate our complex, dynamic environment. Perceiving and interacting with stimuli in the physical world is an essential skill for any biological agent, and for most humans, the people around us represent dynamic stimuli that we encounter and share space with every day. Accounting for other people in our motor planning is critical - not only for keeping our bodies safe but also for engaging in successful and socially appropriate interactions. Accordingly, there must be a complex interplay between social and visuomotor processes that translates to our seemingly effortless co-existence with the physical world and the other people in it. The present research represents an important step toward understanding this phenomenon.

Acknowledgments There are no conflicts of interest to declare. The authors thank research assistants Alex Barone and Nathan Leggett, and thank Lauren Nicholls for creating Fig. 1. This work was supported with an Australian Research Council Discovery Project grant (DP130100541).

\section{References}

Böckler, A., Knoblich, G., \& Sebanz, N. (2011). Giving a helping hand: Effects of joint attention on mental rotation of body parts. Experimental Brain Research, 211(3-4), 531-545. doi:https://doi. org/10.1007/s00221-011-2625-z

Brincker, M. (2015). Beyond sensorimotor segregation: On mirror neurons and social affordance space tracking. Cognitive Systems Research, 34-35, 18-34. doi:https://doi.org/10.1016/j.cogsys.2015. 07.002

Brozzoli, C., Gentile, G., Bergouignan, L., \& Ehrsson, H. H. (2013). A shared representation of the space near oneself and others in the human premotor cortex. Current Biology, 23(18), 1764-1768. doi: https://doi.org/10.1016/j.cub.2013.07.004

Cardellicchio, P., Sinigaglia, C., \& Costantini, M. (2011). The space of affordances: A TMS study. Neuropsychologia, 49(5), 1369-1372. doi:https://doi.org/10.1016/j.neuropsychologia.2011.01.021

Chao, L. L., \& Martin, A. (2000). Representation of manipulable manmade objects in the dorsal stream. NeuroImage, 12(4), 478-484. doi: https://doi.org/10.1006/nimg.2000.0635

Cho, D. T., \& Proctor, R. W. (2010). The object-based simon effect: Grasping affordance or relative location of the graspable part? Journal of Experimental Psychology: Human Perception and Performance, 36(4), 853-861. doi:https://doi.org/10.1037/a0019328

Cho, D. T., \& Proctor, R. W. (2011). Correspondence effects for objects with opposing left and right protrusions. Journal of Experimental Psychology: Human Perception and Performance, 37(3), 737-749. doi:https://doi.org/10.1037/a0021934

Ciardo, F., Lugli, L., Nicoletti, R., Rubichi, S., \& Iani, C. (2016). Actionspace coding in social contexts. Scientific Reports, 6. doi:https://doi. org $/ 10.1038 /$ srep22673

Cléry, J., Guipponi, O., Wardak, C., \& Ben Hamed, S. (2015). Neuronal bases of peripersonal and extrapersonal spaces, their plasticity and their dynamics: Knowns and unknowns. Neuropsychologia, 70, 313-326. doi:https://doi.org/10.1016/j.neuropsychologia.2014.10. 022

Constable, M. D., Kritikos, A., \& Bayliss, A. P. (2011). Grasping the concept of personal property. Cognition, 119(3), 430-437. doi: https://doi.org/10.1016/j.cognition.2011.02.007

Constable, M. D., Pratt, J., Gozli, D. G., \& Welsh, T. N. (2015). Do you see what I see? Co-actor posture modulates visual processing in joint tasks. Visual Cognition, 23(6), 699-719. doi:https://doi.org/10.1080/ 13506285.2015.1078426

Costantini, M., Ambrosini, E., Tieri, G., Sinigaglia, C., \& Committeri, G. (2010). Where does an object trigger an action? An investigation about affordances in space. Experimental Brain Research, 207(1-2), 95-103. doi:https://doi.org/10.1007/s00221-010-2435-8

Creem-Regehr, S. H., Gagnon, K. T., Geuss, M. N., \& Stefanucci, J. K. (2013). Relating spatial perspective taking to the perception of other's affordances: Providing a foundation for predicting the future behavior of others. Frontiers in Human Neuroscience, 7, 596. doi: https://doi.org/10.3389/fnhum.2013.00596 
De Stefani, E., Innocenti, A., De Marco, D., Busiello, M., Ferri, F., Costantini, M., \& Gentilucci, M. (2014). The spatial alignment effect in near and far space: A kinematic study. Experimental Brain Research, 232(7), 2431-2438.

de Vignemont, F., \& Iannetti, G. (2015). How many peripersonal spaces? Neuropsychologia, 70, 327-334. doi:https://doi.org/10.1016/j. neuropsychologia.2014.11.018

Dolk, T., Hommel, B., Prinz, W., \& Liepelt, R. (2013). The (not so) social Simon effect: A referential coding account. Journal of Experimental Psychology: Human Perception and Performance, 39(5), 1248. doi: https://doi.org/10.1037/a0031031

Evans, G. W., Lepore, S. J., \& Allen, K. M. (2000). Cross-cultural differences in tolerance for crowding: fact or fiction? Journal of Personality and Social Psychology, 79(2), 204. doi:https://doi.org/ 10.1037/0022-3514.79.2.204

Evans, G. W., \& Wener, R. E. (2007). Crowding and personal space invasion on the train: Please don't make me sit in the middle. Journal of Environmental Psychology, 27(1), 90-94. doi:https:// doi.org/10.1016/j.jenvp.2006.10.002

Faul, F., Erdfelder, E., Lang, A.-G., \& Buchner, A. (2007). G* Power 3: A flexible statistical power analysis program for the social, behavioral, and biomedical sciences. Behavior Research Methods, 39(2), 175191. doi:https://doi.org/10.3758/BF03193146

Fogassi, L., Gallese, V., Fadiga, L., Luppino, G., Matelli, M., \& Rizzolatti, G. (1996). Coding of peripersonal space in inferior premotor cortex (area F4). Journal of Neurophysiology, 76(1), 141-157. doi:https://doi.org/10.1007/978-94-017-2403-6 4

Freundlieb, M., Kovács, Á. M., \& Sebanz, N. (2016). When do humans spontaneously adopt another's visuospatial perspective? Journal of Experimental Psychology: Human Perception and Performance, 42(3), 401. doi:https://doi.org/10.1037/xhp0000153

Freundlieb, M., Sebanz, N., \& Kovács, Á. M. (2017). Out of Your Sight, Out of My Mind: Knowledge About Another Person's Visual Access Modulates Spontaneous Visuospatial Perspective-Taking. Journal of Experimental Psychology: Human Perception and Performance, 43(6), 1065-1072. doi:https://doi.org/10.1037/ xhp0000379

Frischen, A., Loach, D., \& Tipper, S. P. (2009). Seeing the world through another person's eyes: Simulating selective attention via action observation. Cognition, 111(2), 212-218. doi:https://doi.org/10.1016/j. cognition.2009.02.003

Gerlach, C., Law, I., \& Paulson, O. B. (2002). When action turns into words. Activation of motor-based knowledge during categorization of manipulable objects. Journal of Cognitive Neuroscience, 14(8), 1230-1239. doi:https://doi.org/10.1162/089892902760807221

Gibson, J. J. (1979). The ecological approach to visual perception. Boston: Houghton Mifflin.

Graziano, M. S., \& Cooke, D. F. (2006). Parieto-frontal interactions, personal space, and defensive behavior. Neuropsychologia, 44(6), 845-859. doi:https://doi.org/10.1016/j.neuropsychologia.2005.09. 009

Graziano, M. S., \& Gross, C. G. (1994). Mapping space with neurons. Current Directions in Psychological Science, 164-167. doi:https:// doi.org/10.1111/1467-8721.ep10770670

Grèzes, J., \& Decety, J. (2002). Does visual perception of object afford action? Evidence from a neuroimaging study. Neuropsychologia, 40(2), 212-222. doi:https://doi.org/10.1016/S0028-3932(01)00089-6

Hall, E. T. (1966). The hidden dimension. Garden City, NY: Doubleday.

Hirsch, L., \& Thompson, K. (2011). I can sit but I'd rather stand: Commuter's experience of crowdedness and fellow passenger behaviour in carriages on Australian metropolitan trains. Paper presented at the 34th Australasian Transport Research Forum (ATRF), 28-30 September, 2011, Adelaide, Australia.

Iachini, T., Ruggiero, G., Ruotolo, F., di Cola, A. S., \& Senese, V. P. (2015). The influence of anxiety and personality factors on comfort and reachability space: A correlational study. Cognitive Processing, 16(1), 255-258. doi:https://doi.org/10.1007/s10339-015-0717-6

Iani, C., Baroni, G., Pellicano, A., \& Nicoletti, R. (2011). On the relationship between affordance and Simon effects: are the effects really independent? Journal of Cognitive Psychology, 23(1), 121-131. doi:https://doi.org/10.1080/20445911.2011.467251

JASP Team. (2017). JASP (Version 0.8.1.1) [Computer software]. Retrieved from https://jasp-stats.org/

Jordan, J. S., \& Knoblich, G. (2004). Spatial perception and control. Psychonomic Bulletin \& Review, 11(1), 54-59. doi:https://doi.org/ 10.3758/bf03206460

Kalénine, S., Wamain, Y., Decroix, J., \& Coello, Y. (2016). Conflict between object structural and functional affordances in peripersonal space. Cognition, 155, 1-7. doi:https://doi.org/10.1016/j.cognition. 2016.06.006

Kennedy, D. P., Gläscher, J., Tyszka, J. M., \& Adolphs, R. (2009). Personal space regulation by the human amygdala. Nature Neuroscience, 12(10), 1226-1227. doi:https://doi.org/10.1038/nn. 2381

Lien, M.-C., Gray, D., Jardin, E., \& Proctor, R. W. (2014). Further evidence that object-based correspondence effects are primarily modulated by object location not by grasping affordance. Journal of Cognitive Psychology, 1-20. doi:https://doi.org/10.1080/20445911. 2014.940959

Lloyd, D. M. (2009). The space between us: A neurophilosophical framework for the investigation of human interpersonal space. Neuroscience \& Biobehavioral Reviews, 33(3), 297-304. doi: https://doi.org/10.1016/j.neubiorev.2008.09.007

Maister, L., Cardini, F., Zamariola, G., Serino, A., \& Tsakiris, M. (2015). Your place or mine: Shared sensory experiences elicit a remapping of peripersonal space. Neuropsychologia, 70, 455-461. doi:https:// doi.org/10.1016/j.neuropsychologia.2014.10.027

Maravita, A., Spence, C., \& Driver, J. (2003). Multisensory integration and the body schema: Close to hand and within reach. Current Biology, 13(13), R531-R539. doi:https://doi.org/10.1016/S09609822(03)00449-4

Nicholls, M. E., Thomas, N. A., Loetscher, T., \& Grimshaw, G. M. (2013). The Flinders Handedness survey (FLANDERS): A brief measure of skilled hand preference. Cortex, 49(10), 2914-2926. doi:https://doi.org/10.1016/j.cortex.2013.02.002

Özyürek, A. (2002). Do speakers design their cospeech gestures for their addressees? The effects of addressee location on representational gestures. Journal of Memory and Language, 46(4), 688-704. doi: https://doi.org/10.1006/jmla.2001.2826

Pappas, Z. (2014). Dissociating Simon and affordance compatibility effects: Silhouettes and photographs. Cognition, 133(3), 716-728. doi: https://doi.org/10.1016/j.cognition.2014.08.018

Patané, I., Iachini, T., Farnè, A., \& Frassinetti, F. (2016). Disentangling Action from Social Space: Tool-Use Differently Shapes the Space around Us. PLoS ONE, 11(5), e0154247. doi:https://doi.org/10. 1371/journal.pone.0154247

Perry, A., Rubinsten, O., Peled, L., \& Shamay-Tsoory, S. G. (2013). Don't stand so close to me: A behavioral and ERP study of preferred interpersonal distance. NeuroImage, 83, 761-769. doi:https://doi. org/10.1016/j.neuroimage.2013.07.042

Pezzulo, G., Iodice, P., Ferraina, S., \& Kessler, K. (2013). Shared action spaces: A basis function framework for social re-calibration of sensorimotor representations supporting joint action. Frontiers in Human Neuroscience, 7, 800. doi:https://doi.org/10.3389/fnhum. 2013.00800

Philbeck, J. W., \& Witt, J. K. (2015). Action-specific influences on perception and postperceptual processes: Present controversies and future directions. Psychological Bulletin, 141(6), 1120. doi:https://doi. org/10.1037/a0039738

Prakash, A., \& Rogers, W. A. (2015). Why some humanoid faces are perceived more positively than others: Effects of human-likeness 
and task. International Journal of Social Robotics, 7(2), 309-331. doi:https://doi.org/10.1007/s12369-014-0269-4

Richardson, M. J., Marsh, K. L., \& Baron, R. M. (2007). Judging and actualizing intrapersonal and interpersonal affordances. Journal of Experimental Psychology: Human Perception and Performance, 33(4), 845. doi:https://doi.org/10.1037/0096-1523.33.4.845

Riggio, L., Iani, C., Gherri, E., Benatti, F., Rubichi, S., \& Nicoletti, R. (2008). The role of attention in the occurrence of the affordance effect. Acta Psychologica, 127(2), 449-458. doi:https://doi.org/10. 1016/j.actpsy.2007.08.008

Rizzolatti, G., Fadiga, L., Fogassi, L., \& Gallese, V. (1997). The space around us. Science, 277(5323), 190. doi:https://doi.org/10.1126/ science. 277.5323 .190

Saccone, E. J., Churches, O., \& Nicholls, M. E. R. (2016). Explicit spatial compatibility is not critical to the object handle effect. Journal of Experimental Psychology: Human Perception and Performance, 42(10), 1643-1653. doi:https://doi.org/10.1037/xhp0000258

Sambo, C. F., \& Iannetti, G. D. (2013). Better safe than sorry? The safety margin surrounding the body is increased by anxiety. Journal of Neuroscience, 33(35), 14225-14230. doi:https://doi.org/10.1523/ JNEUROSCI.0706-13.2013

Sebanz, N., Bekkering, H., \& Knoblich, G. (2006). Joint action: Bodies and minds moving together. Trends in Cognitive Sciences, 10(2), 7076. doi:https://doi.org/10.1016/j.tics.2005.12.009

Sebanz, N., Knoblich, G., \& Prinz, W. (2003). Representing others' actions: Just like one's own? Cognition, 88(3), B11-B21. doi:https:// doi.org/10.1016/S0010-0277(03)00043-X

Soper, W. B., \& Karasik, R. (1977). Use of spatial cues with regard to the invasion of group space. Psychological Reports, 40(3 suppl), 11751178. doi:https://doi.org/10.2466/pr0.1977.40.3c.1175

Stoet, G. (2010). Sex differences in the processing of flankers. The Quarterly Journal of Experimental Psychology, 63(4), 633-638. doi:https://doi.org/10.1080/17470210903464253

Strauss, B. (2002). Social facilitation in motor tasks: A review of research and theory. Psychology of Sport and Exercise, 3(3), 237-256. doi: https://doi.org/10.1016/S1469-0292(01)00019-X

Surtees, A., Apperly, I., \& Samson, D. (2016). I've got your number: Spontaneous perspective-taking in an interactive task. Cognition, 150, 43-52. doi:https://doi.org/10.1016/j.cognition.2016.01.014

Symes, E., Ellis, R., \& Tucker, M. (2005). Dissociating object-based and space-based affordances. Visual Cognition, 12(7), 1337-1361. doi: https://doi.org/10.1080/13506280444000445

Szpak, A., Loetscher, T., Churches, O., Thomas, N. A., Spence, C. J., \& Nicholls, M. E. (2015). Keeping your distance: Attentional withdrawal in individuals who show physiological signs of social discomfort. Neuropsychologia, 70, 462-467. doi:https://doi.org/10. 1016/j.neuropsychologia.2014.10.008
Szpak, A., Nicholls, M. E., Thomas, N. A., Laham, S. M., \& Loetscher, T. (2016). "No man is an island": Effects of interpersonal proximity on spatial attention. Cognitive Neuroscience, 7(1-4), 45-54. doi:https:// doi.org/10.1080/17588928.2015.1048677

Tajadura-Jiménez, A., Pantelidou, G., Rebacz, P., Västfjäll, D., \& Tsakiris, M. (2011). I-space: The effects of emotional valence and source of music on interpersonal distance. PLOS ONE, 6(10), e26083. doi:https://doi.org/10.1371/journal.pone.0026083

Teneggi, C., Canzoneri, E., Di Pellegrino, G., \& Serino, A. (2013). Social modulation of peripersonal space boundaries. Current Biology, 23(5), 406-411. doi:https://doi.org/10.1016/j.cub.2013.01.043

Tipper, S. P. (1985). The negative priming effect: Inhibitory priming by ignored objects. The Quarterly Journal of Experimental Psychology. A, 37(4), 571-590. doi:https://doi.org/10.1080/14640748508400920

Tsai, C.-C., Kuo, W.-J., Jing, J.-T., Hung, D. L., \& Tzeng, O. J.-L. (2006). A common coding framework in self-other interaction: Evidence from joint action task. Experimental Brain Research, 175(2), 353362. doi:https://doi.org/10.1007/s00221-006-0557-9

Tucker, M., \& Ellis, R. (1998). On the relations between seen objects and components of potential actions. Journal of Experimental Psychology: Human Perception and Performance, 24(3), 830-846. doi:https://doi.org/10.1037/0096-1523.24.3.830

Tucker, M., \& Ellis, R. (2001). The potentiation of grasp types during visual object categorization. Visual Cognition, 8(6), 769-800. doi: https://doi.org/10.1080/13506280042000144

Tversky, B., \& Hard, B. M. (2009). Embodied and disembodied cognition: Spatial perspective-taking. Cognition, 110(1), 124-129. doi: https://doi.org/10.1016/j.cognition.2008.10.008

Ulrich, R. (1996). Does immediate arousal enhance response force in simple reaction time? The Quarterly Journal of Experimental Psychology: Section A, 49(4), 972-990. doi:https://doi.org/10. 1080/713755672

Wetzels, R., Matzke, D., Lee, M. D., Rouder, J. N., Iverson, G. J., \& Wagenmakers, E.-J. (2011). Statistical evidence in experimental psychology: An empirical comparison using $855 \mathrm{t}$ tests. Perspectives on Psychological Science, 6(3), 291-298. doi:https:// doi.org/10.1177/1745691611406923

Witt, J. K., Linkenauger, S., \& Wickens, C. (2015). Action-specific effects in perception and their potential applications. Journal of Applied Research in Memory and Cognition doi:https://doi.org/10. 1016/j.jarmac.2015.07.008

Witt, J. K., Sugovic, M., \& Taylor, J. E. T. (2012). Action-specific effects in a social context: Others' abilities influence perceived speed. Journal of Experimental Psychology: Human Perception and Performance, 38(3), 715. doi:https://doi.org/10.1037/a0026261

Zajonc, R. B. (1965). Social facilitation. Science, 149(3681), 269-274. doi:https://doi.org/10.1126/science.149.3681.269 